\title{
DE L'ÉVOLUTION DES USAGES en particulier ET DES BESOINS entre parenthèses
}

A BORDER UN THÈME AUSSI VASTE ne pouvait se faire qu'en structurant ses différents aspects. Les nombreuses communications qui s'y rapportent (17) ont ainsi pu être regroupées selon le découpage "classique» calqué sur les différents types d'utilisateurs finaux de l'eau : les ménages (bien que, on le verra un peu plus loin, il ne faille pas systématiquement assimiler consommation domestique et consommation ménagère), l'industrie (productrice ou non d'électricité), l'agriculture, mais aussi les transports et le tourisme, le tout se trouvant synthétisé en final dans des tentatives d'approche systémique des aménagements. Bien sûr, ces sous-thèmes donnent lieu à des approches soit quantitatives, soit qualitatives suivant les auteurs, de même que si certains sont appréhendés à travers une vision " historiciste ", d'autres sont développés sous l'angle prospectif à travers notamment la mise au point de modèles. Enfin, certaines communications font le parallèle avec la situation des PVD et analysent les solutions possibles en matière de coopération Nord-Sud.

\section{Satisfaire les consommations domestiques « à tout prix » ?}

Vu l'importance croissante que prend la pression de l'opinion publique en matière de problèmes d'environnement (ne serait-ce qu'en termes électoraux...), il est logique que les responsables attachent chaque jour un peu plus d'attention à satisfaire ses attentes. On a bien vu, en France, avec la sécheresse, l'ampleur des conflits sur l'eau qui peuvent résulter d'une insatisfaction, ou du moins d'une incompréhension, du public. Mais ce type de tensions est exacerbé dans les pays du Sud, où l'eau est un enjeu beaucoup plus vital encore. C'est pourquoi cette première partie analysant la consommation domestique sous ses différents aspects est tout à fait d'actualité.

Ménages, industrie, agriculture, transports, tourisme... 
Assurer la quantité, au nord comme au sud

A chacun

son système de mesures

\section{Au nord}

J. Bustarret et M. Bos proposent un suivi quantitatif des tendances générales d'évolution de la consommation domestique, en insistant sur les différents facteurs limitants que ce soit dans les pays industrialisés ou dans les pays en développement.

En préambule, ils soulignent que, contrairement à ce qu'en pense le sens commun, la consommation domestique dans la conception qu'en ont les distributeurs d'eau comprend en plus de la consommation des ménages, celles des petits commerces et des ateliers. Cela introduit d'emblée un biais non négligeable dans les comparaisons car ces consommations sont éminemment variables entre les zones urbaines et rurales (ainsi les consommations urbaines sont logiquement supérieures aux consommations rurales, ne serait-ce que par la présence plus importante de commerces et d'ateliers), mais aussi entre les régions (si les résultats sont très variables, il apparaît clairement que les plus fortes consommations se trouvent en été dans les régions les plus ensoleillées mais aussi dans le Bassin parisien, et ce pour la même raison, l'afflux touristique très exigeant en eau) et plus encore entre les pays où elles ne sont pas forcément comptabilisées de manière similaire (en France, par exemple, c'est le ministère de l'Agriculture qui fournit tous les cinq ans des statistiques stabilisées mais chaque pays a son propre système déjà différent au niveau du compteur du particulier).

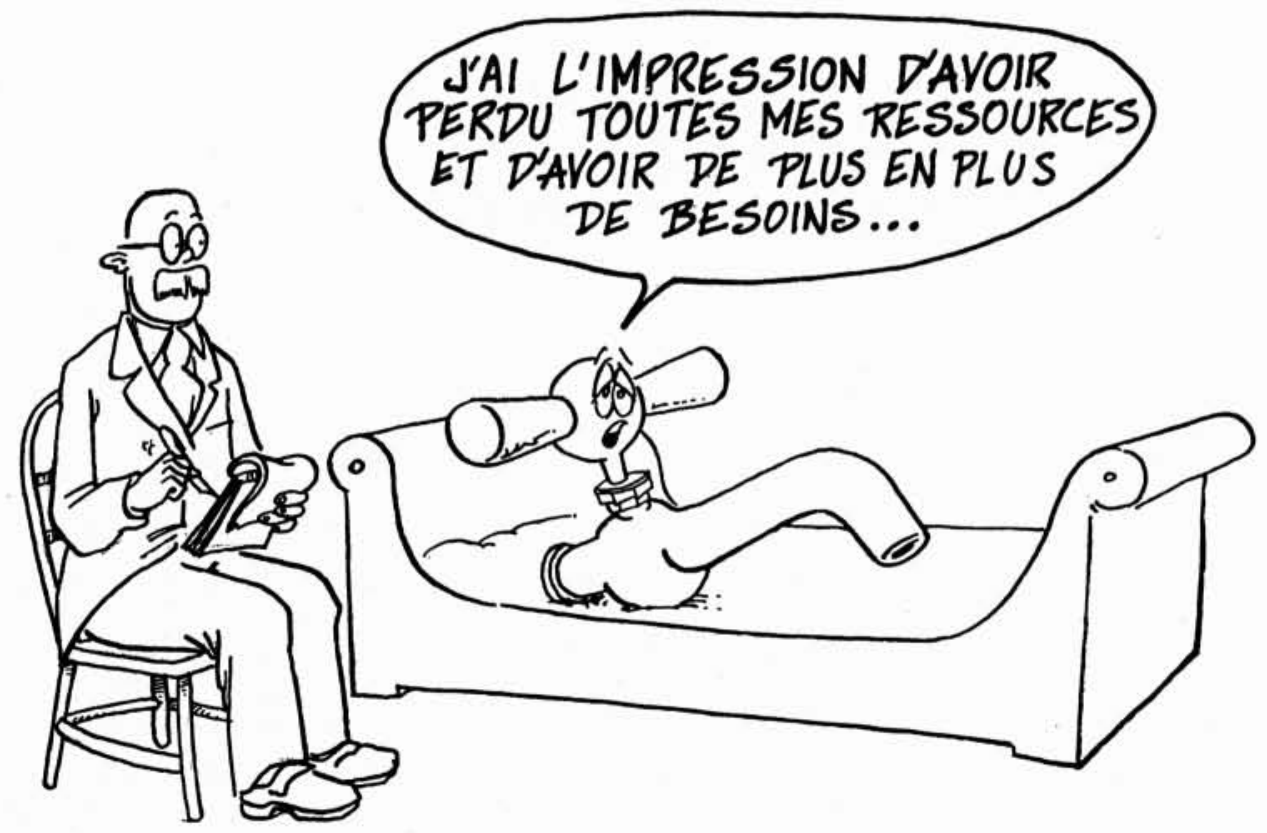


Quoi qu'il en soit il est possible de classer les pays européens en quatre grands groupes en fonction de la consommation journalière moyenne par habitant: moins de $130 \mathrm{l} / \mathrm{j}$ : Belgique ; 130170 l/j : Autriche, France, Grande-Bretagne, RFA, Espagne, PaysBas ; 170-199 l/j : Russie, Luxembourg, Norvège ; plus de $200 \mathrm{l} / \mathrm{j}$ : Italie, Suisse.

En dehors des pays européens, les États-Unis et le Japon présentent des situations particulières de consommation excessive (plus de $300 \mathrm{l} / \mathrm{j}$ ) dans le premier cas par gaspillage, et dans le deuxième, du fait des traditions de propreté (vestimentaire notamment).

En détaillant les différents facteurs limitant de la consommation domestique (nature du logement, nombre de personnes par logement, niveau de vie et d'équipement sanitaire, habitude de consommation et sensibilisation à l'environnement, prix de l'eau), les auteurs estiment qu'en France elle devrait rester plutôt stable encore pendant longtemps, et n'être en définitive influencée que par l'accroissement démographique qui pourrait le faire passer des 3 milliards de $\mathrm{m}^{3}$ de 1990 à 3,3 milliards de $\mathrm{m}^{3}$ en 2010.

\section{Au sud}

En ce qui concerne les pays en développement, l'implication de plus en plus inévitable des grands organismes financiers internationaux (Banque mondiale, Fonds d'aide à la coopération...) dans leurs projets d'adduction d'eau potable nécessite l'élaboration de plans directeurs sur 15 à 20 ans, lesquels doivent prévoir les évolutions probables des consommations et les différentes possibilités d'équilibre des futures sociétés distributrices.

Actuellement, bien que l'alimentation des grands centres urbains soit le plus souvent assurée par des organismes publics, seuls 20 à $30 \%$ de la desserte sont directement issus du branchement sur le réseau, le reste provenant de bornes-fontaines, de revente "sauvage", voire de puits ou de rivières. Si l'importance de cette revente privée prouve que l'usager est prêt à consacrer une part importante de son budget à son alimentation en eau potable, et donc qu'il y a une certaine réserve de capacité de financement domestique exploitable pour équilibrer les comptes des futures sociétés distributrices, cela impose aussi une contrainte : que le prix public soit inférieur au prix des revendeurs, soit en moyenne $5 \%$ du revenu des ménages.

Une politique tarifaire adaptée, notamment avec le développement d'un "branchement social », est donc la clé de l'extension des réseaux publics d'Aep dans les pays en voie de développement. C'est un enjeu important si l'on tient compte qu'en additionnant toutes les consommations induites, la consommation domestique représente en moyenne 55 à $60 \%$ de la consommation totale de ces pays. 
Merci Pasteur

Des réseaux, notoirement incontinents

\section{L'exigence qualitative française au sein de l'Europe}

D. Tricard et A. Montiel se sont penchés sur le rôle des normes sanitaires en matière de santé publique en France, au travers des différentes modalités de leur expression, avec comme toile de fond la transcription en droit français des directives européennes sur le sujet datant de 1980. Le décret 89.3 du 3 janvier 1989 tient en effet compte des recommandations de la directive 80-778/CCE du 15 juillet 1980 mais y intègre les recommandations émises par l'OMs en 1984, sans pour autant dénaturer la spécificité réglementaire française.

Ainsi les règles sanitaires peuvent s'exprimer sous forme d'obligations de résultats (valeurs-seuils à ne pas franchir pour 60 paramètres), ou de moyens (périmètre de protection, qualité de l'eau et traitement minimum, agrément du ministère de la Santé sur les types de traitement, désinfection des ouvrages après travaux), sans pour autant négliger l'obligation de qualité jusqu'au robinet, qui constitue le principal «challenge» du distributeur (nécessité de normes beaucoup plus strictes que celles prescrites par la législation afin de maîtriser l'ensemble des processus de l'entrée à la sortie du réseau).

Pour faire respecter ces règles, la puissance publique peut user de recommandations (circulaires administratives, par exemple) ou édicter des obligations (articles de loi) suivant leur degré d'importance. Le contrôle s'exerce alors par le biais de procédures d'autorisations préalables ou d'enquêtes.

En cas de dépassement des normes, suivant la gravité de la pollution consécutive, on interrompra la distribution ou on la limitera à certains usages non dangereux, si on ne décide pas de ne rien faire (pollution vraiment bénigne).

\section{Faire des économies : un problème de réseaux, mais aussi d'équipement sanitaire chez les particuliers}

La mobilisation, la potabilisation, le transport et la distribution d'une eau, même abondante et gratuite dans le milieu naturel, lui confèrent un coût qui justifie de la gérer efficacement. M. Dargent et M. Retkowsky analysent dans leur article la nature et l'importance des pertes du réseau public d'AEP (adduction d'eau potable) qui nuisent à son efficacité, et qui sont soit des fuites soit des volumes consommés non comptabilisés (par exemple, par «bricolage » du compteur chez les particuliers).

La lutte contre ces pertes n'est justifiée que si les dépenses qu'elle occasionne sont notablement inférieures au coût des fuites. D'après diverses études, c'est toujours le cas lorsque le rendement du réseau est en dessous de $75 \%$, mais, en milieu urbain, cela s'avère rentable même lorsque le rendement avoisine $90 \%$ du fait du coût de toute amélioration. C'est cette optique qui a guidé l'agence de l'eau Seine-Normandie qui propose dans son sixième 
programme d'intervention une aide au diagnostic sur les réseaux basés sur l'action préférentielle au niveau des zones les plus fuyardes.

On a pu estimer il y a 15 ans en région parisienne que les pertes en réseau se montaient à $40 \%$ dont une moitié sur le réseau de distribution proprement dit et l'autre moitié dans la plomberie des usagers. Dans le bâti urbain, $80 \%$ de ces fuites se localisent chez les usagers domestiques et $20 \%$ chez les collectifs, avec une moyenne de $4 \mathrm{l} / \mathrm{h}$ et par unité sanitaire. Il semble que les écoles primaires soient particulièrement touchées.

Le principal facteur de fuite reste le mauvais état des équipements de wC responsables de $80 \%$ des fuites contre $10 \%$ pour la robinetterie et $10 \%$ pour les conduites.

Un effort important doit donc être fait en matière de qualité de conception et d'exécution des matériels comme des installations, tout en privilégiant l'entretien et la maintenance. Une politique tarifaire incitative devrait pousser au choix de la qualité, de même qu'une information bien diffusée. En dernier lieu, il serait souhaitable que les distributeurs se préoccupent de la pression et de la physico-chimie de leur eau.

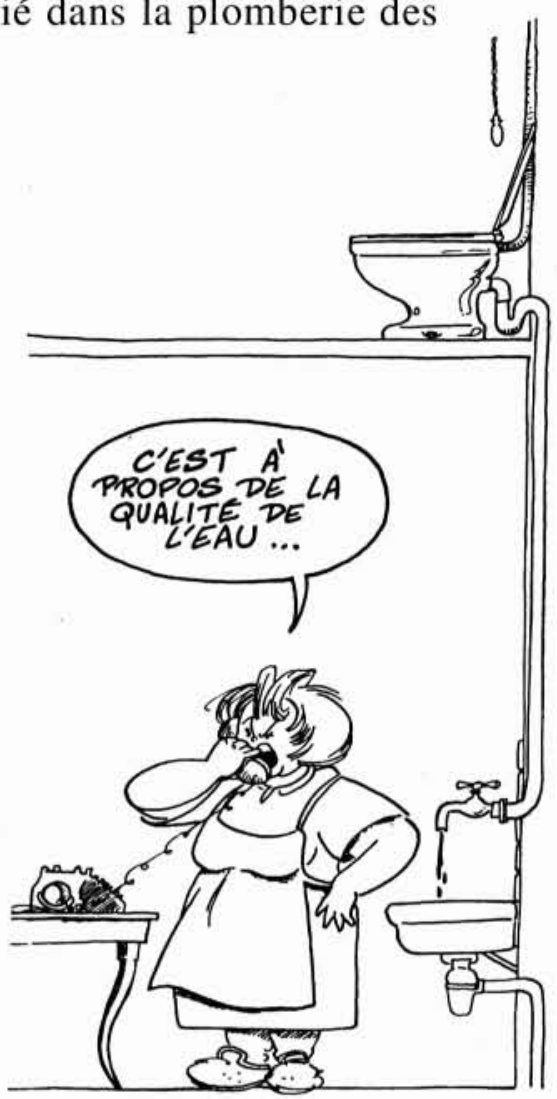

\section{Les consommations industrielles face aux défis de l'environnement}

La «vastitude» de ce sous-thème est bien illustrée par la différence d'échelle entre les deux seules communications qui s'y rapportent : l'une se préoccupe du bassin Seine-Normandie (soit $30 \%$ de la population française) quand l'autre a pour horizon les changements globaux qui risquent de frapper la planète dès le siècle prochain.

\section{En France, nul besoin de s'alarmer}

E. Kepes essaye de déterminer à travers l'exemple du bassin Seine-Normandie sur quels types d'usage de l'eau le décideuraménageur peut espérer avoir le plus de maîtrise du fait d'une 
En France,
on n'a pas
de pétrole...

Eau

et nucléaire

font-ils

la paire? bonne possibilité de prévision de leurs évolutions. Si les prélèvements par les collectivités sont en constante progression et semblent indépendants de toute conjoncture, cela n'est pas le cas des prélèvements pour l'irrigation soumis aux fluctuations climatiques ni des prélèvements industriels dépendants de la conjoncture internationale (cours du pétrole particulièrement). Quoi qu'il en soit, les évolutions de la consommation d'eau industrielle, liée ou non au secteur énergétique, sont dominées par les plus gros consommateurs comme EDF, les raffineries ou les déchetteries.

La synthèse des évolutions montre clairement en tout cas que le volume global des prélèvements sur le réservoir Seine-Normandie a diminué et qu'il n'y a donc pas lieu de s'alarmer quant à une éventuelle saturation des ressources disponibles.

\section{Les atouts de l'hydroélectricité au niveau mondial}

B. Chabot nous rappelle que la décennie 1990 a été celle de la prise de conscience des problèmes globaux (pollutions transfrontières, couche d'ozone, accidents nucléaires majeurs, réchauffement climatique), le tout sur fond de développement inégal. Sur toutes ces questions le secteur énergétique pèse lourdement, et c'est ce qui conduit l'auteur à un plaidoyer pour l'hydroélectricité, une des seules formes de production d'énergie n'aggravant pas l'effet de serre (pas d'émission de gaz).

Après un bref rappel de l'«état de l'art " sur les relations entre $\mathrm{CO}_{2}$, activités humaines et température moyenne du globe (qui sont semble-t-il les trois principales composantes de la dérive anthropogénique de l'effet de serre que l'on pense avoir constatée), l'auteur nous présente les différents scénarios de lutte contre le réchauffement élaborés par le Groupe intergouvernemental sur l'évolution du climat (GIEC). Quatre hypothèses (A, B, $\mathrm{C}, \mathrm{D})$ ont été construites, suivant le degré de volontarisme des politiques de lutte se traduisant, en plus de l'aspect réglementaire, par une place plus ou moins grande et plus ou moins rapidement accordée au nucléaire et aux énergies renouvelables. Toutes conduisent à un réchauffement d'ici l'an 2100, mais seul le scénario D, le plus strict, laisse entrevoir une stabilisation du taux de $\mathrm{CO}_{2}$ à cette époque, après son doublement tout de même, avec une augmentation de $+0,8^{\circ} \mathrm{C}$ en moyenne.

Ce réchauffement, de l'ordre du degré, peut paraître modeste, mais il correspond en fait à une moyenne, ce qui veut dire qu'il sera beau- 


\section{La dérive anthropogénique de l'effet de serre (d'après B. Chabot)}

L'existence d'un " effet de serre " (c'est-à-dire piégeage des radiations solaires directes et réfléchies par l'atmosphère) est une des conditions sine qua non de la vie sur Terre. En son absence, la température moyenne du globe serait en effet de $-18^{\circ} \mathrm{C}$, et toute l'eau serait gelée en permanence. C'est en fait la présence dans l'atmosphère de vapeur d'eau, de dioxyde de carbone $\left(\mathrm{CO}_{2}\right)$ et de méthane $\left(\mathrm{CH}_{4}\right)$ qui permet le maintien d'une température moyenne " naturelle " de $15^{\circ} \mathrm{C}$ et donc le fonctionnement des cycles de l'eau et du carbone.

Ce qui pose problème de nos jours est l'amplification de ce phénomène par contrecoup de la libération accélérée dans l'atmosphère principalement de $\mathrm{CO}_{2}$ mais aussi de $\mathrm{CH}_{4}$ du fait des activités humaines. Depuis la révolution industrielle, le taux de $\mathrm{CO}_{2}$ atmosphérique qui était constant au moins depuis le Néolithique s'est élevé de $30 \%$, passant de 270 ppmv (parties par million en volume) à $350 \mathrm{ppmv}$. Deux facteurs expliquent ce phénomène : la déforestation qui rejette annuellement environ 1,6 Gt (d'équivalent-carbone) par an dans l'atmosphère et surtout l'usage des combustibles fossiles qui en rejettent actuellement 5,15 Gt.

Ce phénomène affecte aussi le méthane - qui bien qu'en quantité moindre que le $\mathrm{CO}_{2}$ dans l'air a un pouvoir " d'effet de serre " plus important en valeur relative - dont les concentrations augmentent à cause des activités agricoles et énergétiques (pertes de gaz naturel).
Par ailleurs l'activité humaine a provoqué l'apparition de gaz à " effet de serre" inconnus, ces oxydes d'azote (NOx) et les "fameux" CFCs (dont la responsabilité est notamment avancée pour expliquer le « trou d'ozone) : ces gaz sont issus des moteurs à explosion, des réfrigérateurs et des mousses isolantes. Al'heure actuelle, ces différents gaz contribuent à ce que l'on appelle la " dérive anthropogénique de l'effet de serre " en proportion de $55 \%$ pour le $\mathrm{CO}_{2}, 15 \%$ pour le $\mathrm{CH}_{4}, 6 \%$ pour le $\mathrm{NO}$ et $24 \%$ pour les CFCs.

Si l'on s'intéresse aux contributions respectives des secteurs d'activités économiques à ce renforcement de l'effet de serre, on a les chiffres suivants pour la décennie 1980: énergie (46\%), déforestation (18\%), agriculture (9\%), CFCs (24\%), divers (3\%).

C'est donc le secteur de l'énergie qui est le $1^{\text {er }}$ responsable de la dérive anthropogénique de l'effet de serre. Or, si on ne modifie pas la tendance, en 2025, sa part sera encore plus lourde, $65 \%$, avec une libération de 9,9 Gt/an. Ce constat alarmiste doit " nous " interpeller puisque, en ne s'intéressant qu'au secteur énergétique, les pays industrialisés qui ne rassemblent que $25 \%$ de la population mondiale sont responsables de $75 \%$ des émissions de gaz à effet de serre de ce secteur ; ce qu'on peut résumer en disant qu'un habitant des pays industrialisés émet 8 fois plus de $\mathrm{CO}_{2}$ par an $(3,12$ t) qu'un habitant des pays en voie de développement $(0,36 \mathrm{t})$ !

coup plus marqué aux latitudes moyennes où l'écoulement risque de diminuer de 40 à $70 \%$. En plus des conséquences prévisibles pour la production hydroélectrique, ce phénomène retentira sur l'extension des zones arides, le volume des précipitations neigeuses, les crues, les catastrophes climatiques et en définitive sur les besoins d'irrigation. Il y a donc lieu d'ores et déjà de penser une planification à long terme des équipements, qui repose sur des études de sensibilité régionales.

En matière de lutte contre le réchauffement, l'absence de solution miracle incite l'auteur à promouvoir deux synergies au niveau planétaire : entre l'hydroélectricité et l'Utilisation rationnelle de l'énergie (URE) et entre l'hydroélectricité et l'écodéveloppement.

La première synergie peut grandement faciliter l'électrification rurale dans les PVD, notamment grâce à la généralisation de récepteurs électriques à très haute efficacité couplée au développement de petites centrales hydroélectriques (comme c'est déjà le

Les microcentrales, ça marche! ... en Chine cas en Chine dans 100 cantons pilotes où sont ainsi économisées 
400000 tonnes de bois de feu par an). La deuxième synergie vise la substitution du bois de feu afin d'enrayer le déboisement exponentiel des forêts du Sud et les graves problèmes d'érosion et de crues qu'il soulève en plus de sa contribution à l'effet de serre. Cet axe pourrait devenir un thème majeur de la coopération NordSud particulièrement adapté aux massifs montagneux peuplés et peu électrifiés (Népal, Madagascar...). Il pourrait, entre autres, bénéficier de la «facilité environnement» du Fonds mondial pour l'environnement, créé en 1990 par le PNUE et géré par la Banque mondiale.

\section{Les prélèvements agricoles sur la sellette}

La succession de sécheresses ces dernières années a mis l'agriculture, l'irrigation surtout, sur la sellette. Il est donc tout à fait intéressant de se pencher sur les rapports qu'entretient l'agriculture avec «son » eau, et surtout sur leur avenir.

\section{Le point de vue du distributeur}

Distribution sous pression

Nos agriculteurs ont de plus en plus soif
Créée en 1960, la Société du canal de Provence (SCP) assure à partir des eaux du Verdon et de la Durance l'irrigation de 57000 ha ainsi que l'alimentation en eau de 110 villes et communes des Bouches-du-Rhône, du Var et des Alpes-de-HauteProvence, et des industries de l'étang de Berre (pétrochimie surtout). C'est donc un des principaux acteurs de l'eau dans le sud-est de la France.

Le premier constat présenté par J.-P. Grisolle, G. Masson, B. Gastoud, J. Bremond et P. Rousset est que, depuis 10 ans, les volumes distribués ont augmenté de $50 \%$ pour atteindre 146 millions de $\mathrm{m}^{3}$ qui vont principalement à l'irrigation et à l'industrie.

L'irrigation, qui se pratique « à la demande » par bornes individuelles, représente quasiment la moitié des volumes distribués et la tendance est encore à l'accroissement grâce au développement de l'irrigation automatique qui conduit à une extension des surfaces irriguées. Par contre on constate une meilleure utilisation des équipements et un étalement de la consommation journalière. A l'heure actuelle, la ScP fournit $60 \%$ des surfaces irriguées et $66 \%$ des débits de sa zone d'intervention.

La progression des économies d'eau chez les industriels induit par contre une stabilité du volume global consommé, en dépit d'une multiplication des clients. Ce n'est pas le cas de la consommation urbaine qui a subi un gros «boom » ces dix dernières années, ce qui n'est pas sans poser de problèmes lors des vacances d'été du fait de la sécheresse et de l'afflux touristique. 


\section{Sécheresse et agriculture : attention}

Pour Y. Merillon et A. Roux, les sécheresses réitérées depuis 1988 ont souligné la fragilité de l'équilibre entre le bon état du milieu et l'utilisation des ressources en eau par l'agriculture intensive, qui passe par une conception raisonnée des aménagements. La vivacité des conflits a témoigné de l'ampleur de l'incompréhension entre usagers, agriculteurs et aménageurs.

Pourtant les volumes prélevés par l'agriculture (4 milliards de $\mathrm{m}^{3} / \mathrm{an}$ ) sont faibles au regard de ceux prélevés par EDF (20 milliards de $\mathrm{m}^{3} / \mathrm{an}$ ), les industries ( 5 milliards de $\mathrm{m}^{3} / \mathrm{an}$ ) et l'AEP ( 6 milliards de $\mathrm{m}^{3} / \mathrm{an}$ ). Mais en consommation nette (soit l'eau réellement soustraite du milieu), l'irrigation représente la moitié de la consommation totale (et jusqu'à $75 \%$ l'été dans le SudOuest).

Alors que les autres consommations ont peu évolué, depuis 20 ans l'irrigation a crû exponentiellement, passant de 500000 ha en 1970 à 1,2 million d'ha en 1989. Le principal bénéficiaire de cette extension est le maïs qui en est responsable de $67 \%$ (soit $50 \%$ des surfaces irriguées totales), car c'est la culture valorisant le mieux l'apport d'eau.

Toutefois, et malgré une grande concentration (11 régions représentent $95 \%$ des surfaces irriguées), la France est en moyenne très loin derrière l'Espagne, l'Italie et la Grèce en ce qui concerne le pourcentage de SAU irriguée, mis à part en région ProvenceAlpes-Côte d'Azur.

L'origine du déséquilibre actuel vient en fait de ce que, récemment, l'augmentation de la consommation agricole a dépassé celle des ressources $\left(+520\right.$ millions de $\mathrm{m}^{3}$ contre +340 millions de $\mathrm{m}^{3}$ entre 1979 et 1988).

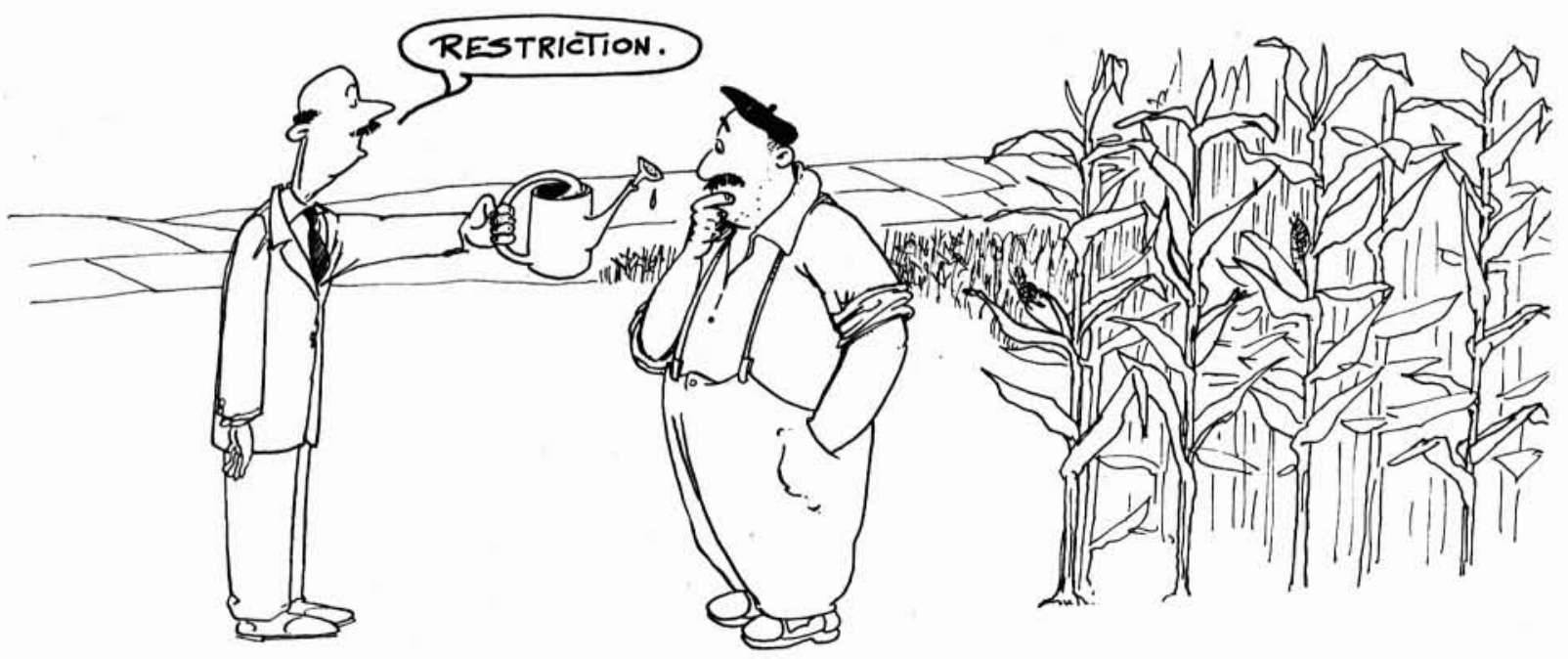




\section{CONSOMMATION}

Consommation et ressources en eau disponibles Irrigations individuelles et collectives

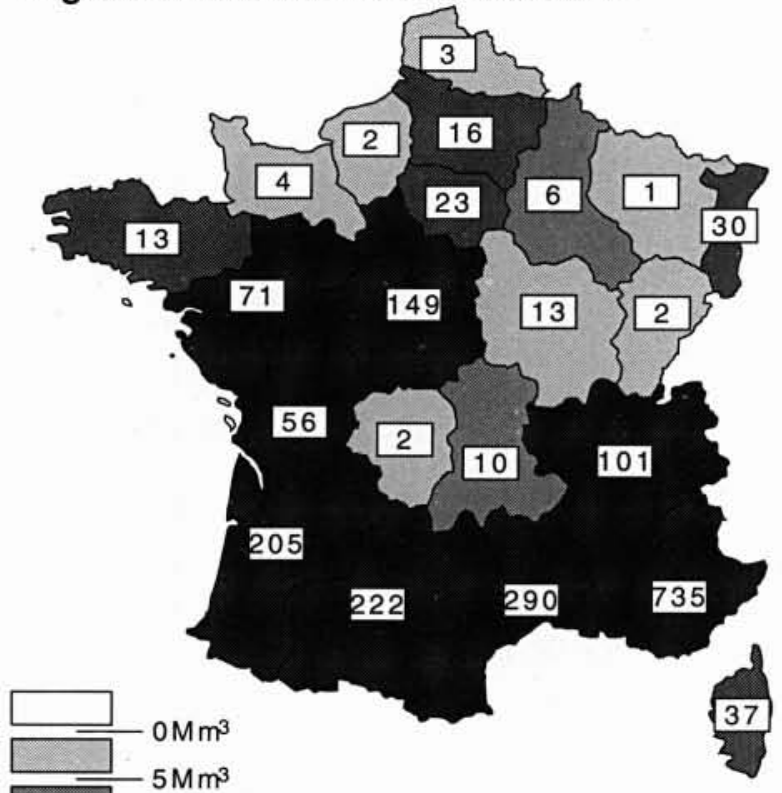

Consommation en 1979 $\left(1990 \mathrm{M} \mathrm{m}^{3}\right)$

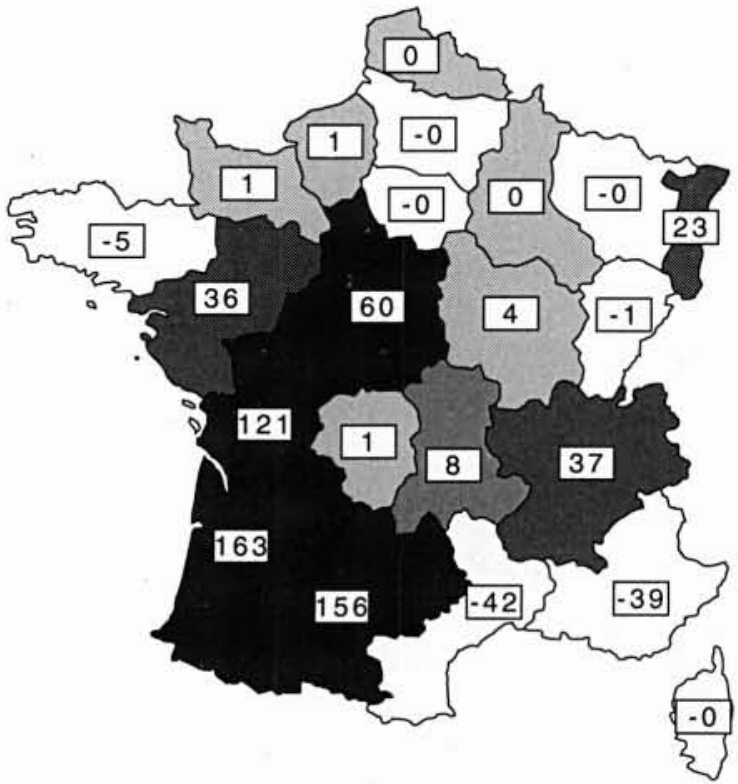

Consommation en 1979-1988 $\left(+520 \mathrm{M} \mathrm{m}^{3}\right)$

\section{RESSOURCES}

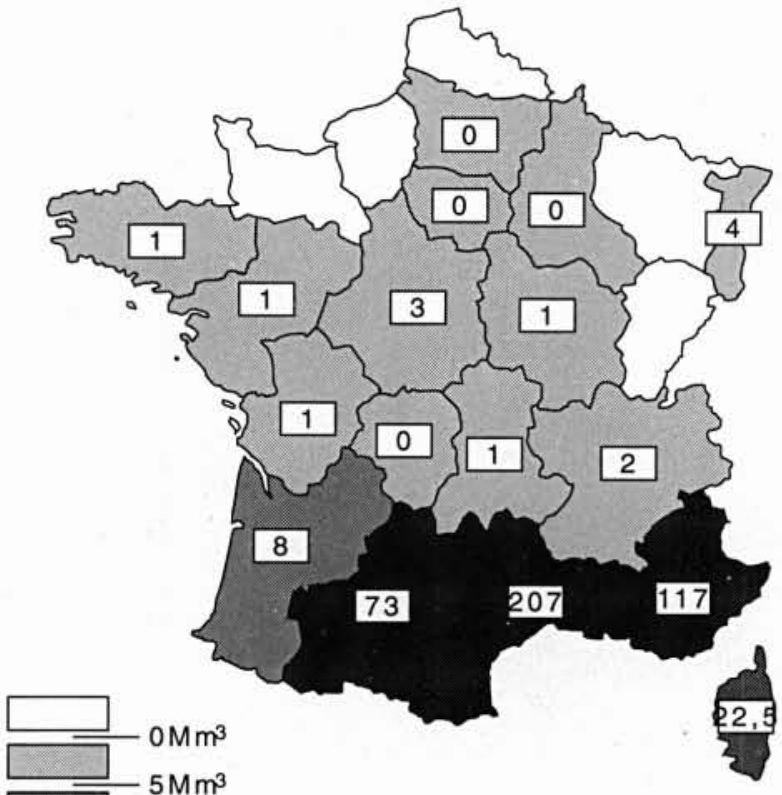

Ressources en 1977 $\left(440 \mathrm{M} \mathrm{m}^{3}\right)$ Hors droits d'eau anciens et convention EDF, CNR

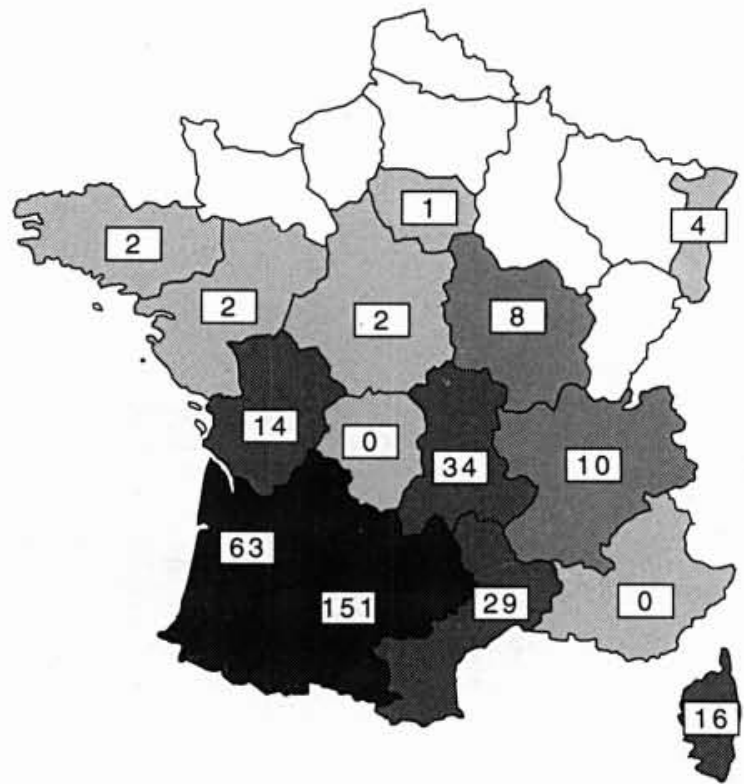

Variation 1977-1988

$\left(+340 \mathrm{M} \mathrm{m}^{3}\right)$

(Investissements collectifs et/ou ayant bénéficié d'une aide directe hors agence de bassin) (Source: Ministère de l'agriculture et de la Forêt-DERF) 
Les auteurs s'attardent ensuite sur les facteurs de développement de l'irrigation, positifs (amélioration de la compétitivité, la sécurité et la qualité), négatifs (contraintes de gestion, assujettissement aux intrants, possibilité de délocalisation) ou difficilement cernables (PAC, GATT).

Un groupe de travail du Cémagref a proposé, à la demande des ministères de l'Environnement et de l'Agriculture, toute une série de mesures aux Assises de l'eau (février 1991) afin d'améliorer les conditions de l'irrigation : développement des techniques économes en eau et formations ad hoc, généralisation des «opérations secteurs de référence irrigation »(OSRI), extension des services d'avertissement et de la régulation automatique, modernisation des vieux réseaux, accroissement de la recherche agronomique et stratégique, amélioration de la planification par des études préalables économiques et écologiques intégrées dans les SAGE (Schémas d'aménagement et de gestion de l'eau), création de commissions locales de l'eau gestionnaires des SAGE.

En conclusion, il ressort bien, qu'en dépit des apparences, le système hydraulique français est d'une grande fragilité.

\section{Irrigation \\ contre hydroélectricité : l'apport de la modélisation}

Ainsi que nous l'expliquent E. Roquero et M. Luque, l'Espagne a un grand besoin de pouvoir estimer les évolutions futures des usages de ses ressources en eau car ceux-ci entrent en compétition avec son importante production hydroélectrique. Ils nous présentent donc une méthode d'évaluation basée, à partir de la télédétection, sur le calcul d'équivalents hydroélectriques des nouvelles surfaces irriguées.

En faisant la différence entre les résultats d'une simulation avec irrigation et d'une autre sans, il est possible d'avoir accès avec une bonne précision à ce type de donnée. L'architecture du système repose sur le modèle HidRA qui maximise la valeur économique de la production hydroélectrique par bassin.

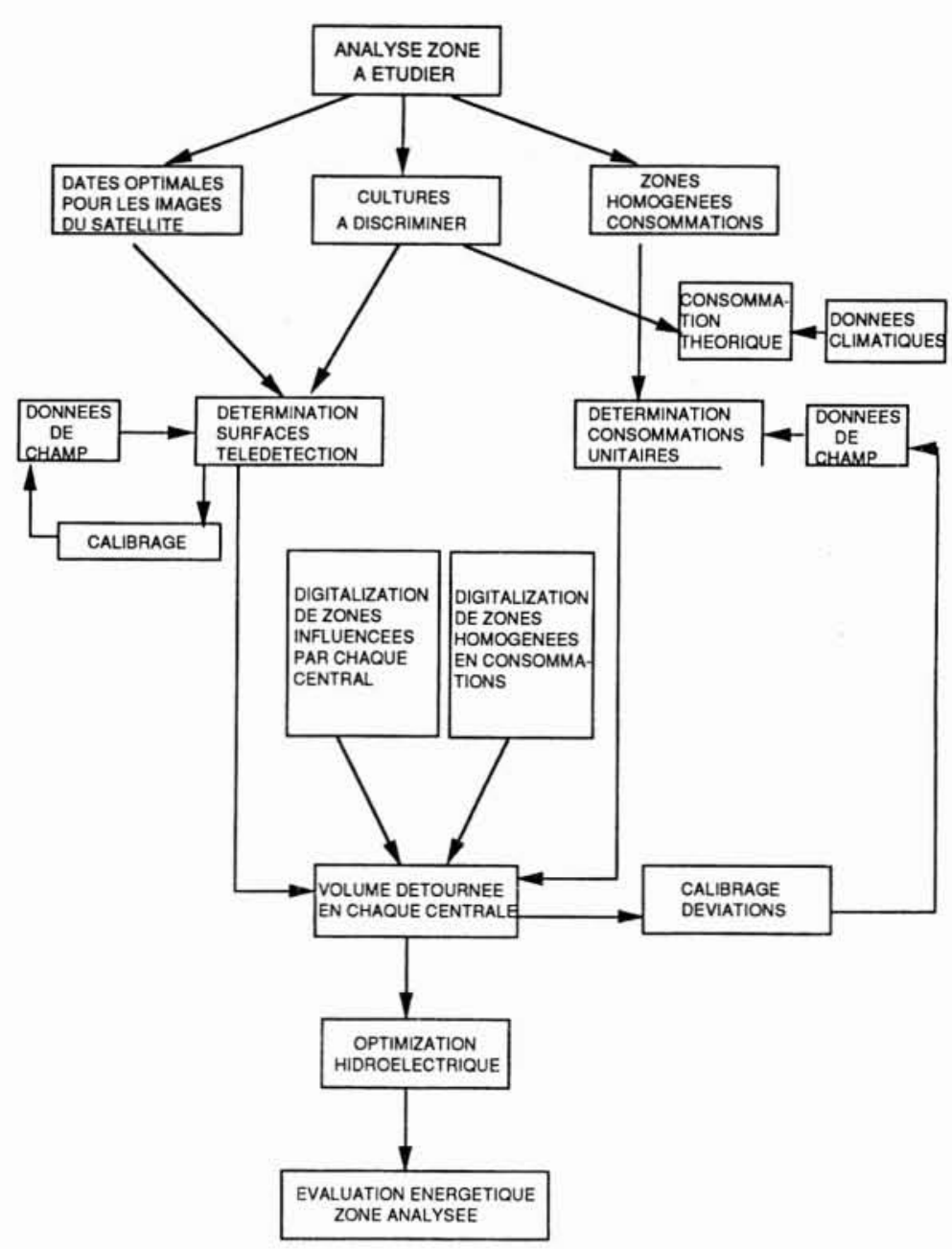




\section{Le transport par voie d'eau en Europe : un « avenir radieux »?}

En retard

d'une guerre, comme toujours...
L'unification européenne et son ouverture à l'Est va entraîner une démultiplication des échanges transfrontières. Face aux multiples difficultés que suscitent les transports routiers ou ferrés (sécurité, impacts...), la voie d'eau est peut-être en bonne place pour retrouver sa «splendeur d'antan » au cœur de l'Europe. En France, il faudra toutefois surmonter de nombreux handicaps.

\section{En France, un réseau d'ampleur mais inadapté}

La France possède un des plus grands réseaux de voies navigables d'Europe $(8500 \mathrm{~km})$. Il souffre malheureusement, selon Ch. Poncet, de son inadaptation aux gabarits actuels, de son absence de reconversion au tourisme et de l'inexistence de connexion entre les réseaux à grands gabarits, au demeurant fort limités $(23,4 \%$ des réseaux alors que l'Allemagne en a plus de $80 \%)$. Handicaps qui trouvent leur origine dans un sous-investissement chronique depuis 25 ans.

De plus, ce réseau inadapté est géré de façon « archaïque » aussi bien au niveau de l'administration que de la batellerie ou des ports. Ainsi, depuis 10 ans, le trafic français a été divisé par deux alors qu'il est resté stable en RFA où il représente neuf fois le nôtre.

Il faut «mettre un bémol » à ce constat pessimiste en ce qui concerne le bassin Rhône-Saône qui est devenu, grâce à la «formule Rhône ", une voie moderne de $300 \mathrm{~km}$ à grand gabarit avec une future extension vers la Bourgogne.

On y compte 3 ports, 26 sites industriels et 5 plates-formes multimodales qui correspondent bien, à un coût très bas, aux attentes des chargeurs et de la flotte qui s'est modernisée. Cette réussite

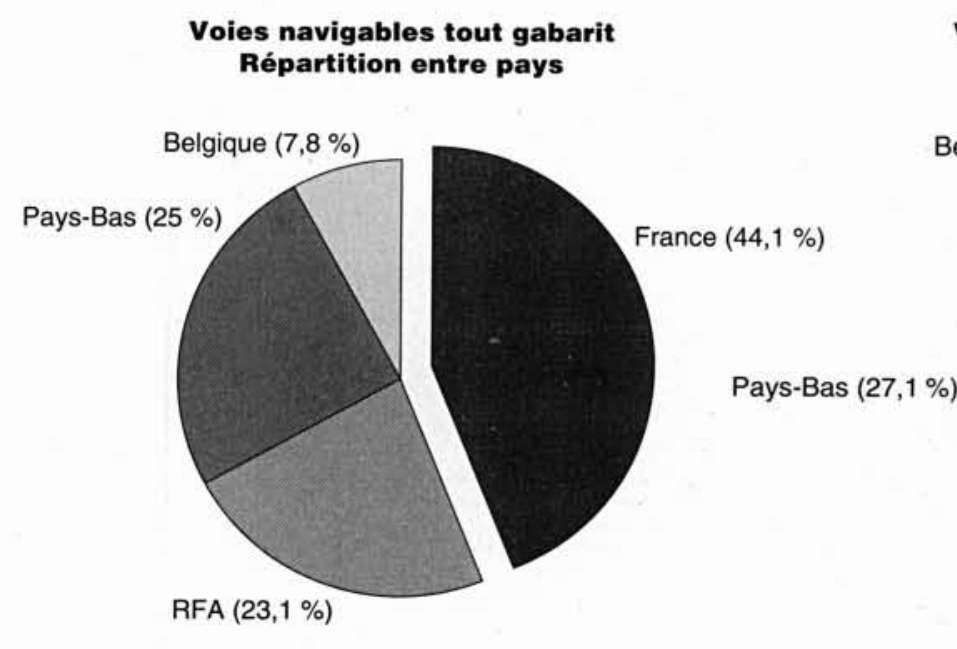
Voies navigables à grand gabarit
Répartition entre pays

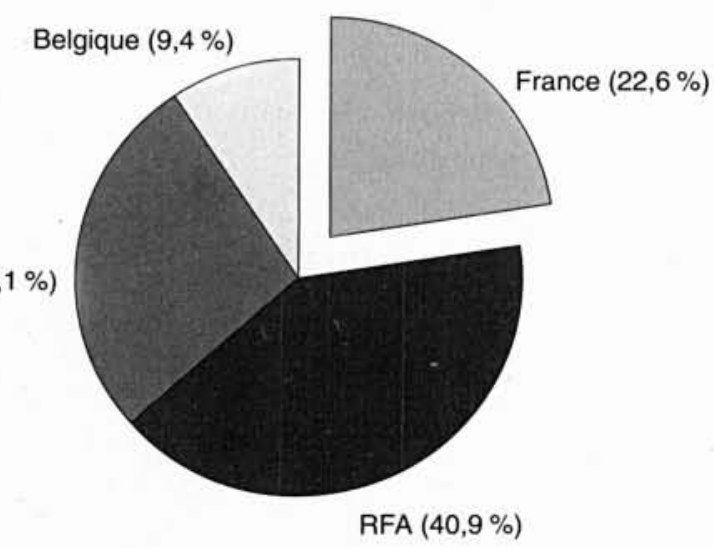


plaide pour un rééquilibrage intermodal des échanges européens au profit d'une liaison Europe du Nord-Méditerranée à grand gabarit.

\section{La voie d'eau dans le Marché unique}

L'Europe compte $19300 \mathrm{~km}$ de voies navigables, nous indique C. Meistermann, mais elle est centrée sur les $1000 \mathrm{~km}$ navigables du Rhin, de ses affluents, de leurs canaux et deltas. La flotte de plus de 15000 bateaux (principalement néerlandais) représente une capacité de 13 millions de tonnes de port, qui est surtout exploitée pour les produits liquides et gazeux (hydrocarbures), pour lesquels elle garantit une très grande sécurité et un coût très faible.

Actuellement, la demande de transport est de 100 milliards de t.km (soit deux fois le trafic marchandise de la SNCF), dont $50 \%$ se font en transfrontière, type de transport où la voie d'eau est pratiquement sans équivalent pour peu que les infrastructures soient adaptées.

Un autre atout de la voie d'eau est le développement des transports de conteneurs et de la technique du Ro-Ro qui permettent d'avoir accès à des marchandises à haute valeur ajoutée.

L'avenir du transport par voie d'eau est plus que jamais, avec l'ouverture du Marché unique, conditionné par la politique de la CEE. Il reste que, pour peu que l'on accepte de faire payer à l'usager tous les coûts du transport (prestations, infrastructure, coûts sociaux externes), la voie d'eau est le mode de transport le moins onéreux, le plus sûr et celui qui couvre le mieux les coûts d'infrastructure tout en générant le moins de coûts sociaux externes.

Même s'il présente des inconvénients du fait d'un maillage trop large, des ruptures de charges, de sa relative lenteur et des contraintes d'hydraulicité, il serait bon au moins que le transport par voie d'eau serve de délestage de la route et du chemin de fer, d'autant plus que les ex-pays de l'Est sont riches en infrastructures naturelles facilement améliorables.

\section{Le tourisme aquatique, mode ou panacée?}

\section{Le point de vue des techniciens : un certain sens du « bien collectif »...}

M.-J. Duffet, G. Soyer, J. Aubague et G. Tratapel nous présentent différentes stratégies d'intégration des contraintes touristiques dans les aménagements de la Compagnie nationale du Rhône (CNR) et d'EDF. 


\section{Exemples de réalisations techniques d'aménagements touristiques liés à l'eau (d'après M.-J. Duffet et al.)}

Pour répondre à la demande croissante en matière de tourisme aquatique, la Compagnie nationale du Rhône et EDF ont dû s'engager très tôt dans une politique volontariste et dynamique d'" ouverture " non prévue initialement de leurs aménagements en direction des loisirs.

La CNR a eu ainsi a gérer des problèmes d'aménagements sur fleuve (courant...) alors qu'EDF a eu à faire face aux difficultés de gestion des niveaux d'eau de ses lacs artificiels.

\section{Les réalisations de la CNR}

- Création d'une halte nautique à forte fréquentation touristique - L'ampleur de la fréquentation des quais du Rhône par les plaisanciers à Avignon a incité la mairie à créer en 1987 une halte nautique de 62 places au pied du Palais des Papes. Pour s'affranchir des contraintes fluviales (crues, courants, corps flottants...) on a disposé en avant de la halte une péniche de $38,5 \mathrm{~m}$ servant à la fois de capitainerie et de " masque flottant ". II a toutefois été nécessaire d'y rajouter un écran flottant de $26 \mathrm{~m}$ installé audessus d'un seuil immergé. Grâce à ce dispositif, les crues de 5 ans n'atteignent plus que des vitesses de $0,5 \mathrm{~m} / \mathrm{s}$ contre $2 \mathrm{~m} / \mathrm{s}$ avant sa mise en place.

- Création d'un complexe nautique intégré à une Zac - La CNR a décidé d'intégrer sa retenue hydroélectrique de Sault-Brenez à la Zac lancée par la commune de Montalieu dans I'Isère. Sur 150 ha, elle propose ainsi une " cascade de loisirs ", au cœur d'un espace de verdure, avec comme équipements : un port et ses pontons pour les plaisanciers, un centre nautique, des plages, un camping et un parc public (tennis, karting, tir, mini-golf,...). S'y rajoutent des croisières et des promenades en petit train.

- Création d'un stade d'eau vive - Le but est de créer une rivière ou d'aménager un cours d'eau existant afin de le rendre apte à la pratique de haut niveau des sports d'eau vive (kayak, raft, hydrospeed), c'est-à-dire lui conférer les caractéristiques d'un torrent (tracés de berges, profil en long, écoulements, obstacles) tout en conservant un aspect le plus naturel possible. Grâce à a participation dès la conception des responsables sportifs, il est possible de valoriser au maximum ce genre d'équipements au plan sportif et économique tout en respectant l'environnement du site.

A Thuringe, près du Rhin, le stade d'eau vive est intégré à un parc multi-activités ludo-spor- tives construit en 1992, le long d'un canal, au cœur de l'agglomération.

Le projet associe un parcours de canoë-kayak, l'aménagement des abords et la réhabilitation des quartiers riverains en leur conférant une vocation touristique. Le parcours de kayak « en dérivation " du canal comprend un bassin d'échauffement dans le bassin d'une écluse, relié par un ouvrage d'alimentation au parcours sportif de $350 \mathrm{~m}$ dont la pente varie de 0,4 à $1 \%$. Cet équipement utilisable presque toute l'année permet la pratique de compétitions comme les entraînements ou l'initiation.

En plus des équipements annexes propres au kayak on a réalisé d'autres structures à vocation plus ludiques (mur d'escalade, piste de skate-board, pistes cyclables, sentiers pédestres, aires de jeux pour enfants,...).

De plus des frayères à poisson ont été aménagées près du canal afin d'accroître l'intérêt écologique de l'ouvrage d'ensemble.

\section{Les réalisations d'EDF}

EDF a aménagé depuis 19534 grands barrages sur le plateau du Levezou près de Rodez (Aveyron) dont celui de Pareloup, le plus important, qui alimente l'usine du Pouget (450 MW). Elle a été vite confronté à une importante fréquentation de cette retenue qui en dépit de son isolement à $800 \mathrm{~m}$ d'altitude attire sur ses $70 \mathrm{~km}$ de berges plusieurs milliers d'estivants français et étrangers qui y pratiquent la natation, la planche à voile, le canoë, la voile, le ski nautique, la pêche, la plongée sous-marine, tout en s'hébergeant en camping ou en bungalow. C'est ainsi que $50 \%$ des visiteurs des lacs du Levezou se retrouvent autour de la retenue de $\mathrm{Pa}$ reloup, dont les structures d'accueil bien qu'insuffisantes (11 hôtels et 10 campings répartis sur 4 communes) recensaient en 1991230000 nuitées. Globalement les retombées économiques sont évaluées à $658 \mathrm{MF}$ ce qui est loin d'être négligeable pour maintenir la région en vie.

Cela a amené EDF, en dépit des difficultés à concilier un niveau d'eau "touristique " avec une bonne gestion économique du réservoir, à passer 120 conventions avec les utilisateurs du domaine concédé et à organiser chaque été le "challenge du sourire" associant activités sportives (hydro-ULM, triathlons, joutes nautiques, aviron, kayak...) et activités culturelles (concerts, expositions...). 
Créées il y a une quarantaine d'années, ces deux sociétés ont vu leur mission initiale (irrigation, transport de l'eau, production d'électricité) débordée par l'essor des loisirs nautiques.

La CNR a eu ainsi à faire face aux problèmes techniques liés à l'intégration de l'usage touristique (2 à 3000 passages en 1991 sur le Rhône) dans des aménagements fluviaux, c'est-à-dire à gérer les problèmes de crues, de courant, de dérive de corps flottants et d'envasement. Elle a ainsi construit sur le Rhône, une trentaine de ports et bases nautiques, allant même jusqu'à créer de toutes pièces des "parcs d'eau vive » propices à la compétition internationale en canoë-kayak (exemple du parc de Thuningue).

EDF, de son côté, gère 150 grands barrages qui représentent 30000 ha de plans d'eau situés dans les zones de piémonts en difficulté où le tourisme aquatique peut être un important facteur de revitalisation. Ce qui l'a poussé à aménager les abords de quelques-uns de ces barrages (exemple du lac de Pareloup) pour l'accueil touristique, malgré les difficultés soulevées par le maintien d'un niveau estival suffisant pour les activités nautiques et la nécessité de vidange périodique pour l'entretien des ouvrages.

A la CNR comme à EDF, c'est une certaine idée du «bien collectif » qui a présidé à ces réalisations.

\section{Le point de vue des gestionnaires : intégrer}

La situation particulière de l'eau dans notre société des loisirs actuelle tient, aux dires de J. Laffont et P. Steinlein, à ce qu'elle constitue à la fois un milieu fragile (donc à protéger) et un élément majeur de détente (donc à aménager).

D'un point de vue écosystémique, tout plan d'eau constitue un patrimoine biologique conditionné par son amont, ses berges et son régime hydraulique.

Mais c'est aussi un élément majeur du paysage propice à la contemplation, à la détente, aux activités de plein air, à la recherche d'une nature authentique...

Pour concilier ces deux approches, il faut veiller à ce que tout aménagement exploite au mieux les potentialités du "pays » et gère finement la ressource en eau. Techniquement, il importe de tenir compte de la géographie locale, de regrouper de façon assez lâche les équipements de façon à différencier les accès à l'eau suivant chaque activité, de doubler les équipements avec des espaces enherbés et boisés, et d'aménager les rives. En bref, d'intégrer.

Une attention particulière sera portée aux plans d'eau marnants en ce qui concerne le respect des berges (frayères) et la qualité de l'eau.

Le respect de l'environnement ne doit cependant pas empêcher toute rentabilité mais conduire à une gestion patrimoniale réglementée entre les différentes contraintes. Alors, le « retour sur investissement » pourra être profitable au milieu naturel. 


\section{Le point de vue du sociologue : de la nature comme « bien de consommation »}

J. Blanchin s'interroge sur le poids des activités récréatives par rapport aux autres utilisations du patrimoine « eau ». C'est selon lui l'incompréhension croissante du citadin vis-à-vis de la nature qui est à l'origine des conflits qui peuvent se créer autour d'un aménagement.

Le développement des activités professionnelles sans contacts physiques avec la nature a fait perdre à l'homme actuel son statut d'acteur du milieu. Par ailleurs, le remplacement du repos dominical par le loisir fait de celui-ci une finalité et le seul moyen de retrouver la nature qui par la force des choses devient un bien de consommation géré par les institutions. A mesure que croît le besoin de liberté, les espaces de liberté se restreignent, d'où les conflits.

Après cette première partie sociologique, l'auteur passe en revue les diverses activités de loisir aquatique et en analyse les évolutions plus ou moins pressenties. Il remarque que les activités traditionnelles comme la pêche ou la chasse sont en perte de vitesse malgré une certaine reprise sous forme de "produits touristiques ». Par contre, on constate une explosion des activités naturalistes et surtout sportives, ciblées (canoë-kayak, hydrospeed...) ou grand public (baignade, voile...). Les activités contemplatrices (plage, tourisme fluvial) sont, elles, toujours aussi importantes.

Ces activités peuvent se répartir en trois groupes : ayant trait à la faune et la flore, nageantes et flottantes, touristiques et de divertissement. Cela permet de mieux cerner les éventuelles incompatibilités ou synergies par rapport à l'espace, aux débits, aux séquelles sur les biotopes, ou aux installations.

Enfin, l'auteur nous présente une expérience de gestion concertée sur le bassin Seine-Normandie : la Communauté des usagers de bord de l'eau (Cube) qui regroupe les partenaires institutionnels, les professionnels du tourisme, les ruraux et les structures de développement. Les premières réalisations ont été la publication d'un livre Arc-en-ciel sur les activités aquatiques et la création d'un observatoire télématique de la qualité de l'eau alimenté par les usagers. 


\section{Vers une approche systémique de l'aménagement des cours d'eau ?}

Afin d'harmoniser les contraintes et les tendances observées dans les précédents sous-thèmes, il est vraisemblable qu'il sera de plus en plus nécessaire de modifier en profondeur nos conceptions souvent trop sectorielles des aménagements. C'est l'objet de cette partie qui, après avoir brossé un tableau général de l'histoire des rapports entre l'homme occidental et ses rivières, détaille diverses solutions techniques ou méthodologiques faisant appel à l'analyse multicritères et à l'approche systémique.

\section{Approche historique : vers le «temps de l'environnement »}

Constatant que chaque civilisation possède sa propre perception des cours d'eau, J.-C. Wolff distingue quatre grandes périodes dans l'évolution de nos rapports avec nos rivières.

Le temps de la dévotion correspond à la civilisation des premiers hommes dont la mystique des sources et des rivières générait une vénération passive.

Avec les premiers villages, monastères et moulins vient le temps de la pénétration et des implantations. Ceux-ci se situent près des sources ou au bord de l'eau qui est utilisée pour la boisson, la pêche, l'abreuvement des troupeaux, l'arrosage des cultures, le transport et bien sûr la protection. Pour l'auteur, c'est le temps de l'équilibre, où les hommes font alliance avec leurs rivières, sans recourir aux aménagements.

C'est la volonté d'exploiter la force hydraulique qui va mener au temps de l'aménagement et de la conquête de l'eau, période qui débute aux alentours du $\mathrm{XII}^{\mathrm{e}}$ siècle et s'étend jusqu'à l'aube des années 1980 ! C'est l'époque des implantations artisanales puis industrielles au fil de l'eau, des tentatives de régularisation des débits, de l'essor de l'adduction d'eau et de la navigation. Malgré des succès évidents (ne serait-ce que par le niveau de développement qu'elle a permis), cette appréhension de nos cours d'eau touche aujourd'hui à ses limites, en grande partie du fait de ses impacts environnementaux.

Cette constatation est à l'origine de la quatrième période recensée par J.-C. Wolff, celle de l'environnement, débutée selon lui il y a moins d'une dizaine d'années et qui se caractérise par le sentiment accru de la valeur patrimoniale et écologique de nos cours d'eau. La rivière se perçoit dorénavant aussi comme un paysage et un milieu vivant. Il s'ensuit la nécessité d'unir les approches mathématiques et naturalistes afin de construire une véritable approche globale des rivières dans leurs bassins versants qui per- 
mettra vraiment d'intégrer les notions d'équilibre instable, d'entretien du milieu et de gestion en partenariat.

\section{Principes de la détermination du débit de garantie biologique (d'après B. Coupry)}

II s'agit de déterminer le débit minimum permettant de garantir les conditions de vie existantes dans un cours d'eau. Différentes étapes sont nécessaires.

\section{Étude cartographique}

A l'aide de descripteurs de terrain, on établit une cartographie caractérisant spatialement les différents habitats et leurs potentialités de production ainsi que leurs relations. La typologie comprend 7 facies : rapide, radier, courant, profond, pool, profond deltaïque et cascade.

\section{Étude hydraulique}

Sur des zones jugées représentatives ou spécialement intéressantes aux plans écologique ou hydraulique, on suit par des mesures de terrain principalement les évolutions des paramètres physiques gouvernant la richesse biologique du milieu, à savoir la hauteur d'eau, la vitesse et la surface mouillée.

\section{Étude physico-chimique}

La qualité physico-chimique est le facteur prédominant en ce qui concerne la richesse biologique. II faut notamment que le débit minimum garantisse le maintien de cette qualité, la dilution des rejets (optimisation du bilan d'oxygène) et la minoration des impacts thermiques (moins de $1^{\circ} \mathrm{C}$ d'augmentation de température).

\section{Étude biologique}

On s'intéresse systématiquement aux 2 principaux groupes de faune aquatique : invertébrés benthiques (insectes, crustacés...) et poissons. Les populations d'invertébrés benthiques sont directement influencées par la vitesse du courant. Celle-ci est répartie en 3 classes ( $<30 \mathrm{~cm} / \mathrm{s}, 30-50 \mathrm{~cm} / \mathrm{s},>50 \mathrm{~cm} / \mathrm{s}$ ) et on re- cherche le débit pour lequel chacune des classes est représentée et recouvre moins de $60 \%$ des vitesses. On compare aussi les valeurs de surfaces mouillées en permanence, seules aptes à accueillir une population normalement dense, avec celles de l'étiage.

Pour les poissons, on différencie les cours d'eau à salmonidés dominants de ceux à cyprinidés dominants. Dans le $1^{\mathrm{er}}$ cas, grâce à la méthode des micro-habitats appliquée à la truite, on détermine l'évolution de la biomasse en fonction du débit. Dans le $2^{\mathrm{e}}$ cas, on utilise plutôt la démarche suivie pour les invertébrés du fait de la diversité des peuplements.

Qualitativement, on cherche le débit maximisant la diversité de l'habitat. Comme les vitesses, on répartit les hauteurs d'eau en 3 classes $(<30 \mathrm{~cm}, 30-80 \mathrm{~cm},>80 \mathrm{~cm})$ et on calcule les écarts-types des répartitions des classes observées pour chaque débit. L'inverse du produit des écarts-types des hauteurs et des vitesses représente l'indice de diversité des écoulements (IDE). Quand il est maximum, on a le débit garantissant le meilleur équilibre. Quantitativement on évalue les surfaces pondérées utiles en fonction de I'IDE.

Enfin on s'intéresse spécifiquement aux frayères remarquables et on s'assure que le débit minimum n'empêche pas la circulation du poisson.

\section{Synthèse}

Même si toutes les valeurs de débit trouvées satisfont du point de vue hydraulique à toutes les exigences des communautés biologiques en place, on prendra comme valeur du débit de garantie biologique la plus forte des valeurs calculées.

\section{Approche biologique : garantir les débits}

Une des premières conséquences de la prise en compte de la nature de milieu vivant d'une rivière est la nécessité d'intégrer dans un aménagement respectueux un "débit de garantie biologique »(DGB). B. Coupry le définit comme étant le débit minimum n'entraînant pas de risque environnemental pour un cycle hydrologique complet, c'est-à-dire garantissant au moins les conditions de vie existantes. 
Sa détermination fait appel à une méthode multicritères reposant sur l'analyse cartographique, hydraulique, physico-chimique et biologique ; le but étant de déterminer un débit minimum pour chacun des paramètres étudiés, le DGB étant alors la plus grande des valeurs calculées.

\section{Approche organisationnelle : pour des modèles globaux}

L'approche systémique de problèmes complexes - comme ceux que pose la diversité des demandes pesant sur les aménagements suppose, pour être efficace, une organisation conceptuelle sans faille mêlant rigoureusement « sectoriel » et « transdisciplinaire ».

Le Groupe de recherche Rhône-Alpes sur les infrastructures et l'eau (GraIE), auxquels appartiennent les auteurs (B. Chocat, F.-N. Cres, G. Hubert, P. Le Gauffre), a fait de ces questions un de ses pôles de travail à travers la publication de cahiers techniques et la mise au point d'un prototype de système multi-experts.

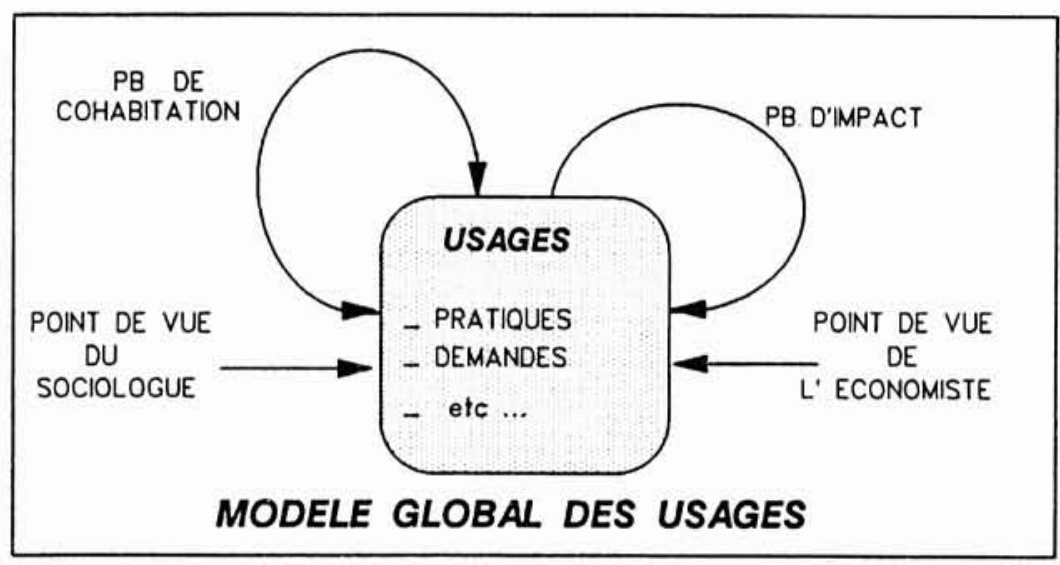

Grâce à des bases de connaissances (sectorielles, transdisciplinaires, d'exemples), il est possible de construire pour chaque site, à l'aide de ces cahiers, trois modèles selon qu'on s'intéresse aux usages, aux diagnostics et aux actions. Il est notamment fourni une grille des problèmes types de cohabitation ou d'impact, ainsi que des alternatives possibles.

Parallèlement, I'InSA de Lyon a élaboré une maquette de système multi-experts qui, en dépit de son intérêt, s'avère trop coûteux. Le GraIE s'oriente maintenant vers des systèmes informatiques moins ambitieux d'aide à l'aménagement.

SCHEMA $n^{\circ} 1$

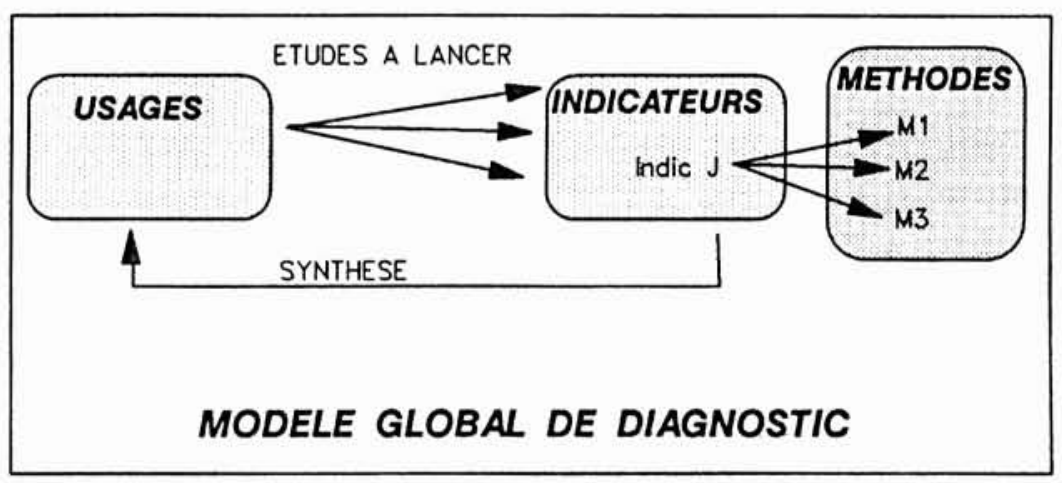

SCHEMA $n^{\circ 2}$

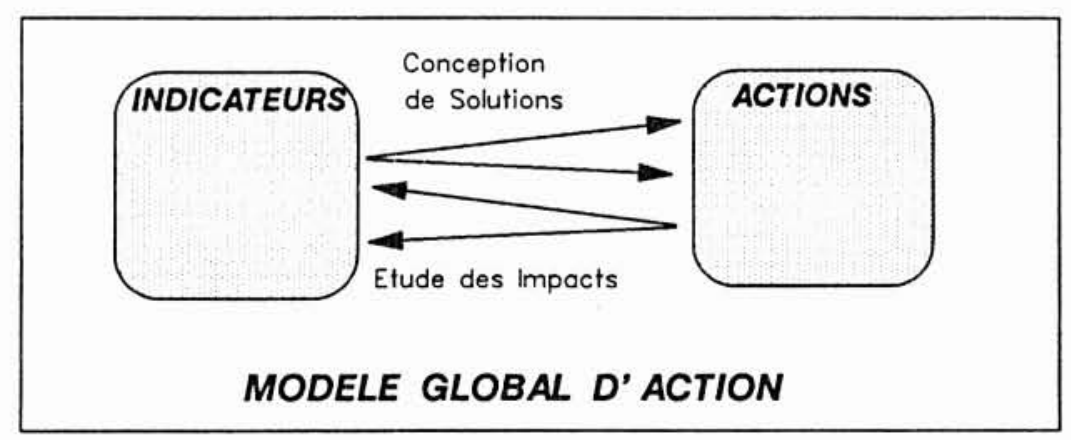

SCHEMA $n^{\circ} 3$ 


\section{Approche économique : évaluer les impacts sociétaux}

C'est bien connu, dans notre société tout est économique... Tout nouvel aménagement se doit donc de tenir compte de cette contrainte. F. Guerber nous montre, dans son article, l'apport de l'évaluation économique dans le choix de scénarios ayant trait à la construction de nouveaux barrages à vocation multiple en amont de Paris.

En effet, les quatre grands barrages, édifiés dans les années 1950 sur le bassin de la Seine afin d'assurer l'AEP et la protection contre les crues de la région parisienne, se révèlent désormais insuffisants. Avec l'aide de la Sogreah et de son modèle «Barbara », il a été construit quatre scénarios suivant la localisation éventuelle des futurs aménagements (Yonne amont: 4 barrages, AuxerreAthie : 2 barrages, Hauterive-Bois de Cure : 2 barrages, Lac de Champ : 1 barrage).

Évaluer, selon la méthode coûts-avantages, l'impact économique de ces aménagements a soulevé le problème de la comptabilisation des biens non marchands comme les coûts environnementaux notamment. La solution a consisté à évaluer les bénéfices à partir

\section{Principes, méthodes de l'évaluation économique appliquée aux aménagements (d'après F. Guerber)}

La méthode utilisée est celle des coûts-avantages. Issue de la théorie économique néo-classique, elle permet de comparer des projets divers mais se heurte à l'estimation monétaire des biens non-marchands, associés à tout projet d'aménagement hydraulique, comme l'adduction d'eau potable, la navigation fluviale, la protection de l'environnement par le soutien des étiages, l'irrigation ou l'utilisation industrielle de l'eau.

On peut cependant estimer les bénéfices au regard des coûts évités en matière de crue, de pénurie d'eau,... On construit donc différents scénaries qui permettent de comparer les différents coûts moyens annuels (CMA) évités, et ainsi d'estimer par différence les bénéfices annuels. Actualisés sur 50 ans, ceux-ci sont comparés aux investissements prévus pour chaque scénario.

\section{Estimation des coûts dus aux inondations}

On estime les coûts de submersion infligés à chaque type d'occupation du sol déterminée, en fonction de la probabilité d'occurrence de la cure et de son extension. Ces résultats basés sur un certain nombre de points sont extrapolés à l'ensemble du bassin.

\section{Estimation des coûts pour l'AEP}

C'est le produit, en cas de rupture de I'AEP, du nombre d'habitants touchés par le coût journalier pour chacun et par le nombre de jours de la défaillance.

\section{Estimation des bénéfices pour l'irrigation}

Peu approfondie, cette question se résume à comparer les rendements du maïs en sec et du maïs irrigué.

\section{Estimation des bénéfices \\ liés à la qualité de l'eau}

En soutenant les étiages des rivières, les barrages améliorent la qualité de l'eau et diminuent de ce fait les coûts de traitement des rejets par les stations d'épuration. On peut donc avoir une estimation indirecte de la valeur de la qualité de l'eau.

\section{Critiques}

La principale critique est que tous les calculs sont basés sur des multiplications de moyennes ce qui limite les possibilités d'appréciation des marges d'erreur et ne donne de toute façon accès qu'à des ordres de grandeur. 
des coûts moyens annuels évités (CMA) actualisés sur 50 ans en matière d'inondations, d'AEP, d'irrigation et de qualité de l'eau. On a ainsi obtenu des ordres de grandeur intéressants mais malheureusement basés sur des moyennes.

Quoi qu'il en soit, il en ressort que par rapport aux investissements nécessaires, c'est le scénario «Lac de Champ » qui est le plus favorable. On a aussi pu se rendre compte de l'importance économique de l'AEP et de l'irrigation qui conditionnent en grande partie les plans de financement des ouvrages.

Malgré son imprécision, cette méthode se révèle donc «assez » puissante pour des choix de cette nature.

\section{Conclusion}

Ce tour d'horizon des évolutions probables des différents modes de consommation des ressources en eau nous montre qu'en dépit de la subsistance de points noirs le plus souvent biens connus (problèmes quantitatifs au Sud, qualitatifs au Nord), le «monde de l'eau " possède les moyens de résoudre la majorité des difficultés pressenties pour le futur, que ce soit au plan technique ou au plan conceptuel.

Il reste maintenant, pour que cela puisse se traduire vraiment dans les faits (et entre autres outrepasser les égoïsmes nationaux et financiers), à faire passer le message en profondeur dans le « corps social " afin que celui-ci révolutionne vraiment sa manière de penser l'eau.

Si la gestion patrimoniale semble le seul moyen pour affronter les prochains changements globaux, l'eau, sommet médiatisé et affectif de "l'iceberg " environnemental, est sans doute un des vecteurs privilégiés pour effectuer cette révolution dans nos habitudes de penser le monde. 


\section{Que ressort-il des débats?}

Menaces

sur l'eau:

de Mickey

aux centrales

thermiques
Des «puces»

d'eau?

Gare aux

«miracles»

\section{A propos du rapport général}

- Le rapport général de synthèse de P.-F. Tenière-Buchot a donné lieu à un certain nombre d'échanges. Le premier a concerné le développement des usages récréatifs de l'eau et les risques de «Disneylandisation » de la nature. Puis il a été débattu de la pertinence des échelles territoriales de compétences par rapport à la gestion globale du milieu naturel qui « ignore » bien sûr les limites administratives... Il est vrai qu'en ce qui concerne la gestion de l'eau, la législation a de plus en plus tendance à «coller » aux réalités locales (exemple des schémas d'aménagement et de gestion des eaux, SAGE). L'avenir des nappes phréatiques, soumises à une exploitation croissante et à des risques de pollution accrus, paraît incertain. Diverses solutions sont envisageables : protection "foncière » des zones d'alimentation, traitement, mélange d'eau polluée et d'eau pure, abandon de l'usage potable.

La consommation d'eau de refroidissement dans les centrales électriques ne risque-t-elle pas de grever la ressource en eau vu les nouvelles techniques employées (aéroréfrigérants) et l'augmentation du parc des centrales particulièrement dans les pays méditerranéens du Nord ? Plus généralement, il semblerait plus judicieux de gérer la demande vu le caractère naturellement limité des ressources en eau. C'est un des attraits des comités de bassins français chargés de répartir les ressources entre les différentes demandes. Toutefois certains choix stratégiques peuvent remettre en question ce mécanisme.

\section{A propos des communications}

- La première communication portait sur les transports par voie d'eau, on s'est intéressé à leurs performances et au développement de l'intégration entre la voie d'eau et les autres modes de transport.

- Les méthodes d'économie d'eau potable élaborées par l'agence Seine-Normandie ont suscité quelques réflexions sur la nécessité d'éviter l'amalgame habitat pavillonnaire, collectif ancien et collectif récent. Pourquoi aussi ne pas envisager, à l'instar du secteur électrique, le développement de compteurs d'eau «à puces " qui permettraient une meilleure transparence et un contrôle beaucoup plus efficace ? Des études sont effectivement en cours.

- En dépit de son caractère apparent de «solution miracle » face au réchauffement planétaire, l'hydroélectricité reste une technologie lourde (même si son amortissement s'étale sur 20 ans), et dont les impacts, aussi bien en termes de qualité des eaux, de peuplement de poissons que de gestion des lits majeurs, ne peuvent être sous-estimés. Mais les techniques évoluent dans le bon sens. 
- La politique tarifaire de la Société du canal de Provence a fait l'objet de quelques éclaircissements. La "préférence agricole » qu'elle pratique découle d'une volonté conjointe du conseil d'administration et du ministère de l'Agriculture. Par ailleurs, si la consommation à l'hectare équipé augmente, c'est bien la conséquence directe de l'augmentation des surfaces irriguées à l'intérieur des périmètres.

- La controverse sur la priorité des usages agricoles de l'eau n'a pas été évitée. On s'est en effet interrogé sur les impacts de l'irrigation vis-à-vis des nappes souterraines, patrimoine écologique à préserver comme le spécifie la loi sur l'eau du 3 janvier 1992. Ce débat est bien entendu dominé par le "poids politique » de la profession agricole qui pèse sur les décisions d'aménagement par la Politique agricole commune qui pourrait compromettre la rentabilité de l'irrigation.

- Grâce au contrôle que l'on peut exercer sur les déclarations des agriculteurs, il semble que le calcul prédictif de la consommation agricole pour la planification des équipements destinés à l'irrigation ou à l'hydroélectricité soit effectivement une méthode fiable. L'Espagne l'utilise pour sa planification hydrologique depuis 20 ans.

- A propos des aménagements touristiques réalisés par EDF et la Compagnie nationale du Rhône (CNR), il est clair que le choix de l'usage prioritaire des équipements revient aux politiques. Mais même en accordant la priorité à l'usage touristique il n'est pas envisageable de s'affranchir des contraintes d'entretien des ouvrages comme les vidanges périodiques. Quand à la solution séduisante du maintien d'un niveau constant grâce à un deuxième petit barrage, elle pose le problème du financement. Remarquons que ces problèmes techniques n'affectent pas les aménagements de la CNR.

- La multiplicité des critères de détermination du débit de garantie biologique paraît à certains devoir déboucher sur des empoignades mercantiles entre les différents usagers. Mais en fait il convient de raisonner en termes de facteur limitant et de coût marginal. Par ailleurs la conception de la rivière en tant que milieu aquatique est tout à fait dans l'esprit de la nouvelle législation sur l'eau et des SAGE. Elle est la base d'une gestion patrimoniale de l'eau, même si la légitimité écologique reste encore falote comparée à la légitimité économique.

- Les nouvelles méthodes multicritères de prise de décision concernant les aménagements, exposées aussi bien par l'agence Seine-Normandie que par la société Hydro-M sont symptomatiques de l'évolution des mentalités. On passe du secret à la publicité, de la justification monofonctionnelle à la justification plurifonctionnelle, du financement de l'État au nom d'un «intérêt général » autoritaire et régalien à un cofinancement par les acteurs locaux dans la mesure de leur intérêt. Les agences de l'eau y ont
De la « gravité » en politique

Un tourisme qui nage dans la politique

\author{
Garantie \\ biologique \\ ou garantie \\ économique?
}


joué un grand rôle mais il reste encore à développer les techniques d'évaluation collective et de répartition équitable.

\section{Conclusions du rapporteur P.-F. Tenière-Buchot}

Les communications de ce thème ont surtout porté sur les pays développés en montrant que dans le fond les sciences et les techniques de l'eau avaient réussi progressivement, en 150 à 200 ans, à arriver à une situation très largement satisfaisante dans les pays comme la France. Cela ne veut pas dire qu'il ne reste pas beaucoup de choses à faire, mais ce sont essentiellement des perfectionnements. Le plus gros a été fait : à peu près tout le monde a de l'eau, des sanitaires et peut boire de l'eau sans danger même si de temps en temps le goût n'est pas bon. C'est loin d'être le cas dans la plupart des pays du monde, ce qui constitue une différence gigantesque.

Comment en est-on arrivé là ? D'abord grâce à une grande mâ̂trise technique de l'eau dont on peut se réjouir. Mais si cette condition est nécessaire, elle n'est pas suffisante et on le voit dans des pays où ces connaissances scientifiques existent mais où il manque les moyens pour concrétiser les projets. Dans notre pays, et c'est le cas dans les pays industriels de manière générale, il y a en plus une maîtrise du financement, ce qui fait la différence entre un pays développé et un pays non développé. Elle signifie savoir organiser, savoir programmer - c'est ce que l'on ne trouve pas dans un pays en développement où très souvent une idée est remise en cause au fur et à mesure de la succession des hommes, et d'ailleurs il faut se méfier de cette discontinuité des services publics ou privés - et elle signifie enfin savoir créer. On ne peut pas financer si on ne sait pas créer un climat de confiance entre les usagers : dans un pays comme la France, même si on oppose les usages agricoles ou industriels aux usages domestiques ou touristiques, on arrive à en parler et à trouver des compromis ; c'est d'ailleurs le rôle essentiel des agences de l'eau. Si ces compromis entre les agents qui représentent les divers usages et besoins n'existent pas, tout projet sera remis en cause.

Mais ces succès, relatifs bien sûr, qui ont été obtenus notamment en France, se traduisent aussi par une plus grande complexité dans les relations entre l'homme et la nature. Il y a là un véritable danger parce que cela se traduit par une nature toujours plus artificielle, toujours plus urbaine, n'hésitons pas à le dire, avec une régression du monde rural, une perte de la mémoire et de la culture rurale, et donc de la compréhension de la nature. Cela provoque en quelque sorte une perte d'âme de la politique de l'eau dont il faut se méfier. Un certain nombre de communications dans le cadre de cette question deux l'ont bien souligné.

En guise de conclusion, il me semble qu'il convient pour les ingénieurs et les techniciens, et aussi pour les autres responsables 
de la politique de l'eau, de faire attention à ce point-là en essayant de développer trois valeurs indispensables pour compenser ou au moins amenuiser cette tendance. Premièrement, il faut élargir les compétences techniques à des compétences de sciences économiques et humaines et de sciences de la nature. Deuxièmement, il faut rechercher une grande honnêteté intellectuelle dans la répartition des risques et des coûts. C'est ce dialogue qui manque tellement dans la réalisation des ouvrages dans les pays en développement : il s'agit de traduire les déséquilibres en répartition des charges. Ma grand-mère dans le temps faisait des ouvrages de dame pour résoudre les problèmes de la pauvreté. Un jour, on a inventé la sécurité sociale. On peut la critiquer pour ses déficits permanent, il n'empêche que le montant financier de la sécurité sociale par rapport aux ouvrages de dames est à peu près ce qu'il faut faire pour les pays en développement par rapport à ce qui se fait en ce moment dans des conférences comme Rio. Et le dernier point, la dernière valeur, c'est le développement du sens du bien commun. Je crois que dès l'école primaire, il faut rappeler qu'audelà de lire, compter et écrire, il faut également inculquer ce sens du bien commun.

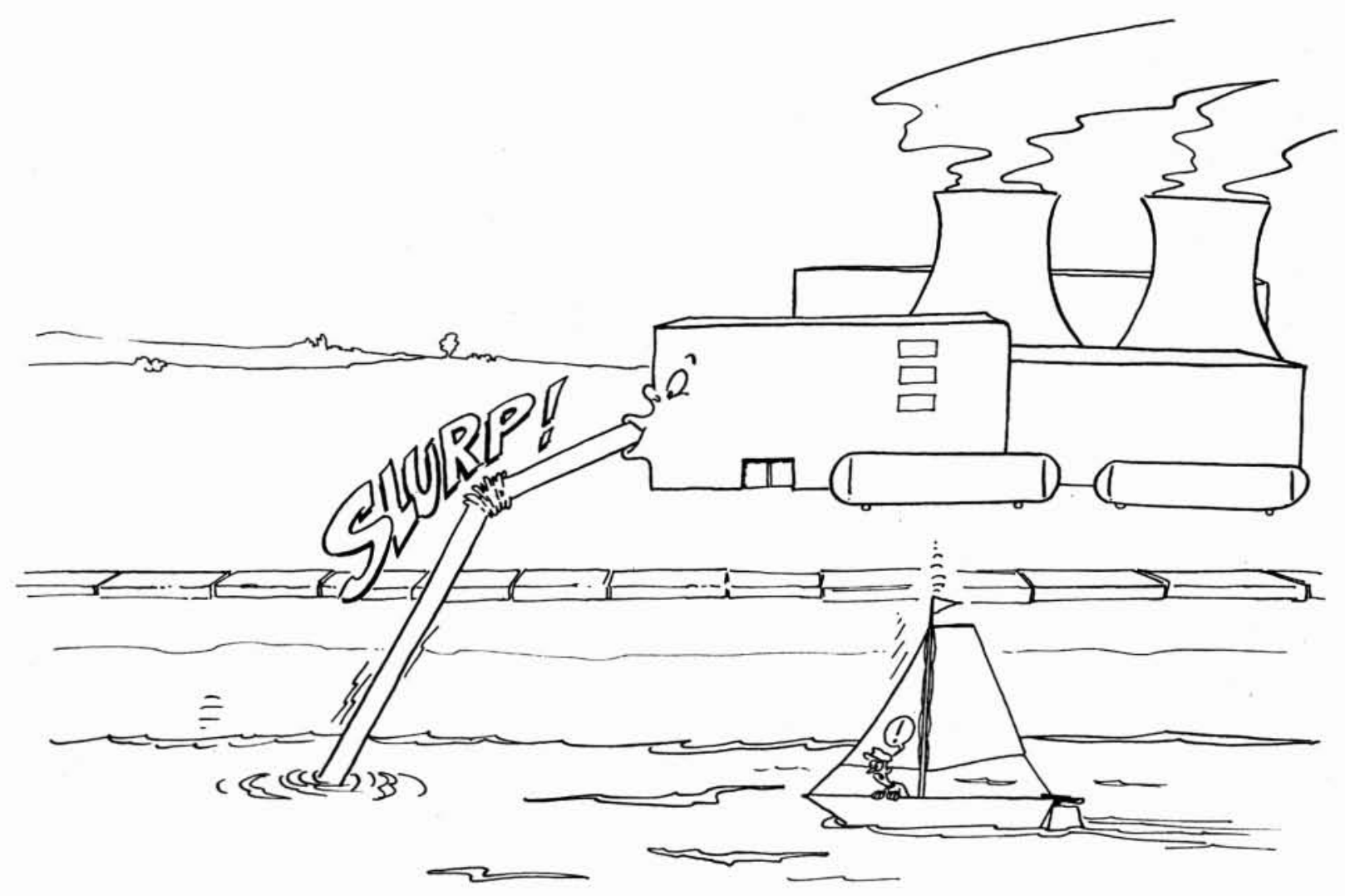




\section{Modélisation des écoulements et gestion prévisionnelle des ressources en eau en Afrique de l'Ouest (d'après E. Servat et al.)}

En Afrique de l'Ouest comme dans le reste des pays en voie de développement, la bonne gestion des ressources en eau est une priorité pour assurer la bonne marche des projets d'aide à la restructuration et à l'intensification agricole comme aux projets d'aménagement hydraulique. C'est l'intérêt des travaux de modélisation de la relation "pluie-débit " qui permettent d'avoir accès à une certaine prédiction de l'évolution des ressources, et autorisent la simulation du fonctionnement des aménagements.

Dans le nord-ouest de la Côte d'Ivoire, l'Orstom mène ces études sur 16 bassins-versants, de 100 à $4500 \mathrm{~km}^{2}$, situés sur les hauts bassins du Niger, du Bandame et du Sassandra. L'objectif de la modélisation est de pouvoir être appliquée par la suite à des bassins versants non jaugés ; grâce à des épurations de prédétermination bâties à partir de variables géomorphologiques, climatiques ou d'occupation du sol.

\section{Modélisation des écoulements}

Deux modèles globaux conceptuels et déterministes ont été utilisés : CREC et GR3. CREC est basé sur un schéma à réservoirs relativement classique articulé sur une fonction de production à 3 paramètres qui donne accès à l'écoulement en prenant en compte la fraction de l'eau précipitée qui y participe et l'évapotranspiration alimentée par l'humidité du sol) et une fonction de transfert à 4 paramètres avec un terme d'écoulement rapide et un autre d'écoulement lent).

GR3 repose sur 2 réservoirs reliés par un hydrogramme unitaire : le réservoir-sol qui commande la répartition de la pluie par le biais de l'évapotranspiration potentielle; un hydrogramme unitaire qui décrit la propagation des débits jusqu'au $2^{\mathrm{e}}$ réservoir ; le réservoir-eau gravitaire qui reçoit les débits routés et alimente ainsi le débit de la rivière selon une loi de vidange quadratique.

Les variables choisies pour l'étude sur 60 années des 16 bassins versants portent sur les caractéristiques de chaque bassin (surface), celles du climat (précipitations) et celles de l'occupation des sols (structures de végétation et des cultures). En dépit de l'utilisation de méthodes statistiques du type ACP, il n'a pas été possible de dégager de relations significatives entre les paramètres et les variables. II apparaît toutefois que l'occupation des sols joue un grand rôle, de même que les précipitations des premiers mois de la saison des pluies qui conditionnent la couverture végétale. On peut noter aussi que GR3 fournit de meilleurs résultats que
GREC du fait d'une meilleure indépendance des paramètres.

En conclusion, en dépit des défaillances citées précédemment, la méthodologie de cette étude (regroupement en " stations-années ", calages annuels systématiques, caractérisation des jeux de paramètres calés et recherche d'équations de prédétermination des paramètres au moyen des techniques de répression multiple) permet d'accéder à des résultats de bonne qualité autorisant le calcul d'apports sur des bassins non jaugés en zone de savane soudanaise, ce qui peut être exploité dans le cadre d'études de projets d'aménagement.

\section{Gestion d'un système d'eau aménagé}

L'instabilité du régime des précipitations dans le nord-ouest de la Côte d'Ivoire pose problème quant aux performances des équipements qui y sont implantés à vocation hydroélectriques ou agricoles. II s'avère donc important en matière de planification de vérifier la réalité d'une telle instabilité. Sur le bassin du Sassandra, I'Orstom a étudié deux séries de 200 ans de données décadaires de pluies et de débits à partir de 5 postes pluviométriques. En étudiant les variations des écarts-types, il a été possible de détecter une année de rupture dans la pluviométrie, l'année 1969. Un modèle de simulation, basé sur des loi des fuites ajustées à chaque décade observée et tirer aléatoirement, a permis de construire la relation pluie-débit à l'aide des autorégressions et de corrélations, qui s'avère tout à fait "plausible". Cela a permis d'alimenter le modèle de simulation de fonctionnement du système d'eau aménagé à vocation d'irrigation et hydroélectrique, qui permet ainsi d'effectuer un bilan complet des entrées-sorties sur la retenue considérée. A l'issue des calculs, on connaît donc l'état de la retenue (la cote du plan d'eau) à la fin du pas de temps.

On constate à travers cette simulation que les apports aussi bien que les divertissements diminuent notablement après 1969 , et ce principalement aux dépens de l'agriculture. II s'avère aussi que les petites retenues n'ont qu'un très faible pouvoir tampon vis-à-vis des défaillances ce qui soulève de graves difficultés pour l'agriculture irriguée qui en dépend souvent.

Enfin, l'étude a permis de montrer que l'aménagement de l'amont du bassin du Sassandra limiterait sérieusement la variabilité des apports annuels. Elle plaide aussi pour la reformulation de la notion de "normes hydrauliques" dont la fixité actuelle handicape la réalisation de nouveaux aménagements. 